\title{
Determination of Pulse Width and Pulse Amplitude Characteristics of Materials Used in Pendulum Type Shock Calibration Device
}

\author{
E. BILGIC* \\ TÜBİTAK Ulusal Metroloji Enstitüsü (UME), Gebze Kocaeli, Turkey
}

\begin{abstract}
Calibration of shock and vibration transducers is performed by using the methods described in ISO 16063-13 and 16063-22 standards, at primary and secondary levels respectively. At secondary level, the devices, generating mechanical shock, are pendulum shock calibrator, dropball shock calibrator, pneumatically operated piston shock calibrator and Hopkinson bar shock calibrator. The main parameters and requirements of the shock calibrator devices, named here, are also given in ISO 16063-22 standard. Pulse width and pulse amplitude are the important parameters in calibration of the shock and vibration transducers. Pulse width and shape depend on the material properties. In this paper the determination of pulse width and pulse amplitude characteristics of materials used in pendulum type shock calibration device, designed in TUBİTAK UME, is presented.
\end{abstract}

DOI: 10.12693/APhysPolA.132.857

PACS/topics: 06.20.Dk, 06.20.fb

\section{Introduction}

Events like falling of an object, car crush or launching of rockets produce high amplitude acceleration, called shock. Resistance of such objects, devices and machines to high shock levels, during their storage and operation, is demonstrated or proven by shock tests. Details of the shock tests are described in relevant national and/or international standards or in the in-house methods. Important part of the test is the test spectrum, applied to the device under test. The confidence of such tests mainly depends on the calibration of the test system and especially on the shock transducers. Therefore shock transducers should be calibrated in the range that covers the scope of demanded tests.

Calibration of shock transducers is performed at two levels. These are the primary level, in accordance to ISO 16063-13 standard [1], and secondary level, in accordance to ISO 16063-22 [2]. Laser interferometric calibration of the shock transducers was investigated by A. Link et al. in 1998, who have reported that the computer simulations and experimental investigations also proved that the peak value and the spectral components of shock-shaped accelerations can be measured with expanded uncertainties of less than $0.2 \%$ [3]

Later on, a detailed review of ISO 5347 and its revised and renumbered version ISO 16063 and traceability for vibration and shock measurements was published by H.-J. von Martens [4]. Application of ISO 1606321 and 22 was introduced by some of the manufacturers [5]. Dynamic calibration of different transducers, such as force and pressure transducers, were investigated

\footnotetext{
*e-mail: eyup.bilgic@tubitak.gov.tr
}

and the results were published, taking care of the applied methods and also of the effects of waveform model $[6,7]$. Pulse shape effect, uncertainties of the calibrations and also filtering of the noise were studied by various authors [8-10]. Recently, pulse width of the mechanical shock signals applied to the transducer was stated in the Technical Protocol of CCAUV Key Comparison, as a mandatory parameter [11].

At primary level, in order to generate shock accelerations, Hopkinson bars are used. At secondary level, the devices generating mechanical shock are pendulum shock calibrator, dropball shock calibrator, pneumatically operated piston shock calibrator and Hopkinson bar shock calibrator. The main parameters and requirements of the shock calibrator devices are given in ISO 16063-22 standard. Pulse width and pulse amplitude are the important parameters in calibration of the shock and vibration transducers. Pulse width and shape depend on the material properties.

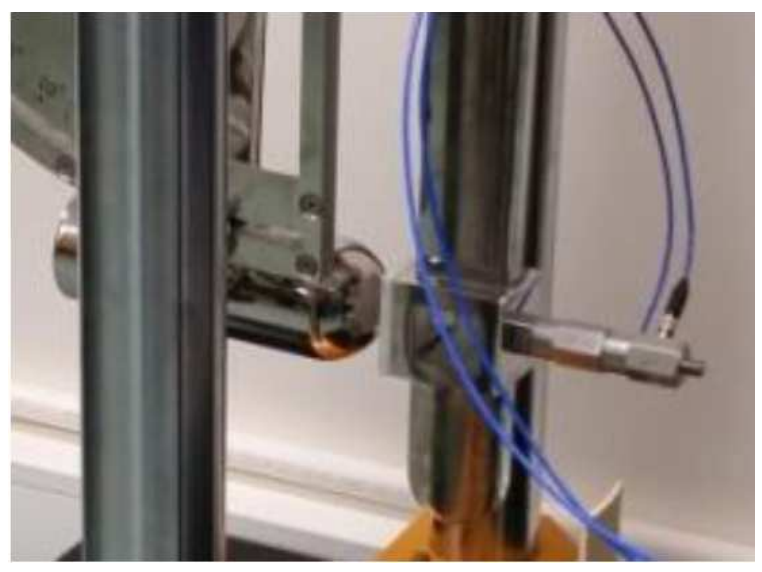

Fig. 1. Hammer and anvil pendulum of shock calibrator device, designed in TÜBİTAK UME. 
A pendulum shock calibrator device was designed in TÜBİTAK UME and manufactured, see Fig. 1. Six different elastic materials (pads) were chosen to use between the hammer pendulum and the anvil pendulum, as shown in Fig. 2. Samples N1 to N4 are made of rubber. Their chemical composition and thicknesses are different, however samples N3 and N4 have the same properties. Modulus of elasticity of the pads was measured in Force Laboratory in TÜBİTAK UME. The results are given in Fig. 3 and in Table I.

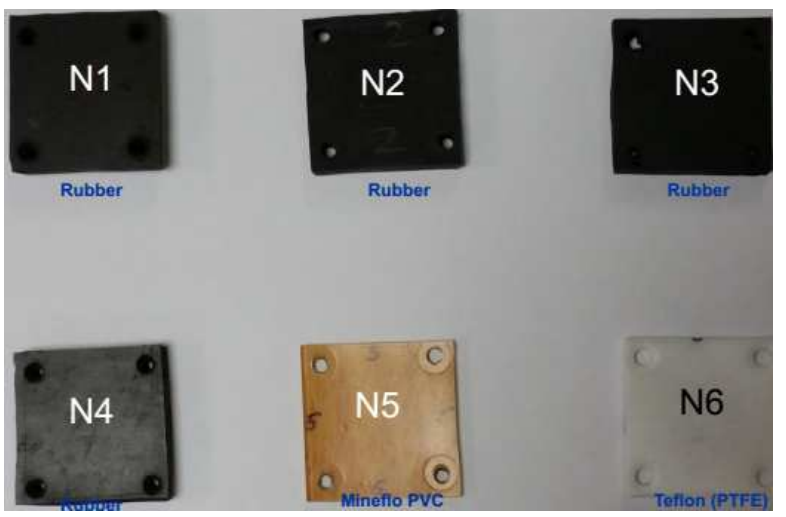

Fig. 2. Pad samples used between hammer and anvil pendulum.

TABLE I

Mechanical properties of pads of the pendulum shock calibrator.

\begin{tabular}{c|c|c|c|c}
\hline \hline $\begin{array}{c}\text { Sample } \\
\text { no. }\end{array}$ & $\begin{array}{c}\text { Surface } \\
\text { area } \\
{\left[\mathrm{mm}^{2}\right]}\end{array}$ & $\begin{array}{c}\text { Thickness } \\
{[\mathrm{mm}]}\end{array}$ & $\begin{array}{c}\text { Modulus of } \\
\text { elasticity } \\
{\left[\mathrm{N} / \mathrm{mm}^{2}\right]}\end{array}$ & $\begin{array}{c}\text { Regression } \\
\text { coefficient } \\
R^{2}\end{array}$ \\
\hline N1 & 1558 & 10.24 & 8.68 & 0.9995 \\
N2 & 1592 & 8.80 & 21.70 & 0.9973 \\
N3 & 1543 & 8.50 & 9.09 & 0.9999 \\
N4 & 1455 & 8.50 & 9.17 & 0.9995 \\
N5 & 1564 & 2.22 & 5.15 & 0.9906 \\
N6 & 1606 & 5.08 & 45.60 & 0.9997
\end{tabular}

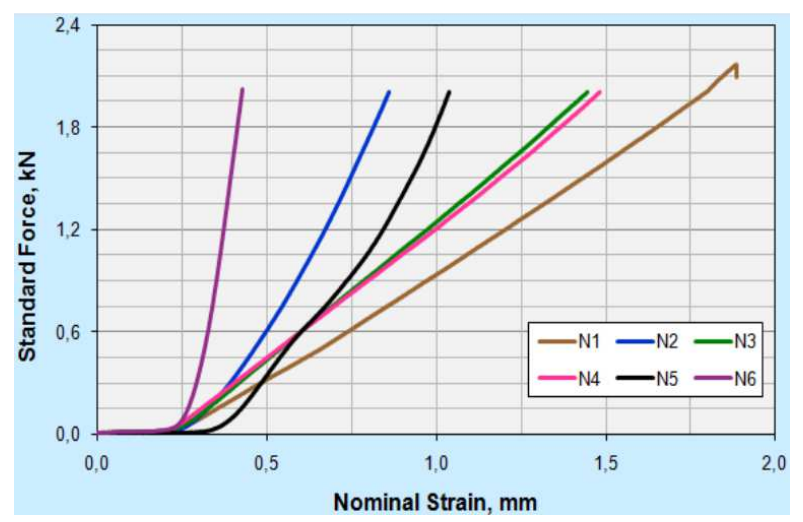

Fig. 3. Stress-strain curves for the pads used between hammer and anvil pendulum.

\section{Determination of pulse width, pulse amplitude and shock sensitivity}

Shock pulse width and shock pulse amplitude of the signal, produced by pendulum shock calibrator, were determined for various drop angles. The drop angle is an angle between vertical axis, parallel to the anvil pendulum, and the tip of the hammer pendulum.

Sample pad N1 and two shock transducer PCBs of type 301A12, in a back-to-back configuration, were mounted on the anvil pendulum. Outputs of the transducers were connected to Agilent digital oscilloscope through four channel signal conditioner PCB of type $482 \mathrm{C}$. One of the transducers was used as reference (Ref.) transducer and the other was the device under test (DUT). Drop angle was changed from $5^{\circ}$ to $15^{\circ}$ and the output signals of the transducers were recorded.

Waveforms of the output signals from signal conditioner for sample N1 are presented in Fig. 4. Output signals for sample N2 to N6 were recorded by repeating the steps described above.
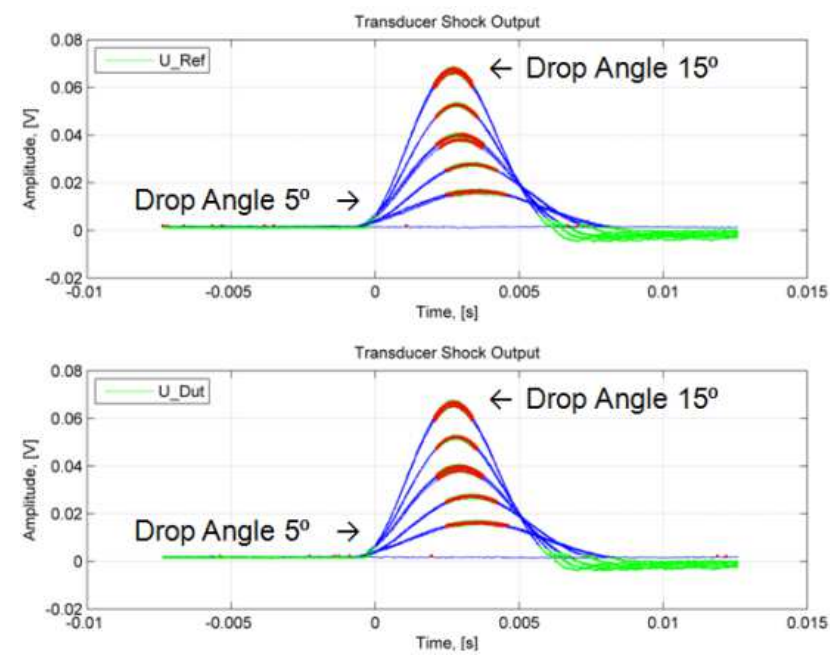

Fig. 4. Waveforms of the output signals from signal conditioner for sample N1.

A calculation code was developed based on Matlab. This code was used to draw the signal output graphs and the calculations and fitting for the pulse width output signals and shock sensitivity of DUT. Sensitivity of the DUT is defined in ISO 16063-22 standard as given below:

$$
S_{\text {dut }}=S_{\text {ref }} \frac{u_{\text {dut, peak }}}{u_{\text {ref, peak }}},
$$

where $S_{\text {dut }}$ is sensitivity of the transducer under test, $S_{\text {ref }}$ is sensitivity of the reference transducer, $u_{\text {dut, peak }}$ is maximum peak value of output of the transducer under test and $u_{\text {ref, peak }}$ is maximum peak value of output of the reference transducer.

In order to determine peak value and therefore the sensitivity of the transducers, three methods are defined. 
Version 1: Selection of maximum values as peak values: Maximum peak value is selected from the series of filtered transducer outputs.

Version 2: Polynomial approximation (time domain): The series exceeding the $90 \%$ (second choice $95 \%$ ) of the peak values are approximated with a parabola and then maximum peak value is calculated.
Version 3: Sensitivity calculation using FFT analysis: Applicable if the complex sensitivity of the reference transducer is known.

In this investigation, Version 1 and Version 2 of the method were used in the calculations. Results for sample N1 are given in Table II.

TABLE II

Signal outputs (pulse amplitude), pulse width and shock sensitivity of DUT for the sample N1.

\begin{tabular}{|c|c|c|c|c|c|c|c|}
\hline $\begin{array}{l}\text { Drop angle } \\
\quad \text { [deg] }\end{array}$ & $\begin{array}{c}\text { Average } \\
\text { ref. output } \\
{[\mathrm{mV}]}\end{array}$ & $\begin{array}{c}\text { Repeatability } \\
\text { ref. output } \\
{[\mathrm{mV}]}\end{array}$ & $\begin{array}{c}\text { Repeatability } \\
{[\%]}\end{array}$ & $\begin{array}{c}\text { Drop angle } \\
\text { [Deg] }\end{array}$ & $\begin{array}{c}\text { Average } \\
\text { pulse width } \\
{[\mathrm{ms}]}\end{array}$ & $\begin{array}{c}\text { Repeatability } \\
{[\mathrm{ms}]}\end{array}$ & $\begin{array}{c}\text { Repeatability } \\
{[\%]}\end{array}$ \\
\hline 5 & 16.37 & 0.15 & 0.89 & 5 & 13.72 & 1.35 & 9.84 \\
\hline 7.5 & 27.70 & 0.06 & 0.23 & 7.5 & 7.23 & 0.02 & 0.31 \\
\hline 10 & 39.59 & 0.41 & 1.05 & 10 & 6.43 & 0.03 & 0.51 \\
\hline 12.5 & 52.71 & 0.08 & 0.14 & 12.5 & 5.98 & 0.01 & 0.20 \\
\hline 15 & 67.04 & 0.35 & 0.52 & 15 & 5.59 & 0.01 & 0.18 \\
\hline $\begin{array}{l}\text { Drop angle } \\
\quad \text { [deg] }\end{array}$ & $\begin{array}{c}\text { Av. peak } \\
\text { sensitivity } \\
{\left[\mathrm{mV} /\left(\mathrm{m} / \mathrm{s}^{2}\right)\right]}\end{array}$ & $\begin{array}{l}\text { Repeatability } \\
{\left[\mathrm{mV} /\left(\mathrm{m} / \mathrm{s}^{2}\right)\right]}\end{array}$ & $\begin{array}{c}\text { Repeatability } \\
{[\%]}\end{array}$ & $\begin{array}{c}\text { Drop angle } \\
\text { [deg] }\end{array}$ & $\begin{array}{c}\text { Average fit } \\
\text { sensitivity } \\
{\left[\mathrm{mV} /\left(\mathrm{m} / \mathrm{s}^{2}\right)\right]}\end{array}$ & $\begin{array}{l}\text { Repeatability } \\
{\left[\mathrm{mV} /\left(\mathrm{m} / \mathrm{s}^{2}\right)\right]}\end{array}$ & $\begin{array}{c}\text { Repeatability } \\
{[\%]}\end{array}$ \\
\hline 5 & 0.0470 & 0.0004 & 0.93 & 5 & 0.0471 & 0.0004 & 0.82 \\
\hline 7.5 & 0.0465 & 0.0002 & 0.36 & 7.5 & 0.0465 & 0.0002 & 0.37 \\
\hline 10 & 0.0469 & 0.0001 & 0.30 & 10 & 0.0470 & 0.0001 & 0.28 \\
\hline 12.5 & 0.0467 & 0.0001 & 0.20 & 12.5 & 0.0468 & 0.0001 & 0.11 \\
\hline 15 & 0.0465 & 0.0001 & 0.15 & 15 & 0.0465 & 0.0000 & 0.08 \\
\hline
\end{tabular}

The results for all samples N1-N6 are presented in Figs. 5-7, for the pulse amplitudes, pulse widths and shock sensitivities respectively.

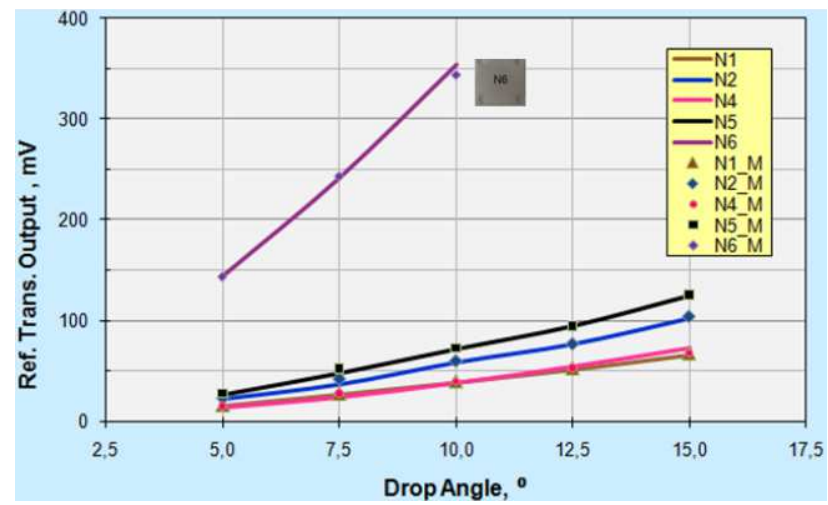

Fig. 5. Pulse amplitudes of ref. transducer and signal conditioner vs. drop angle for samples N1-N6.

\subsection{Discussion}

- The pulse amplitude and pulse width from the shock transducer and signal conditioner depend on the type of the pad mounted on the anvil pendulum.

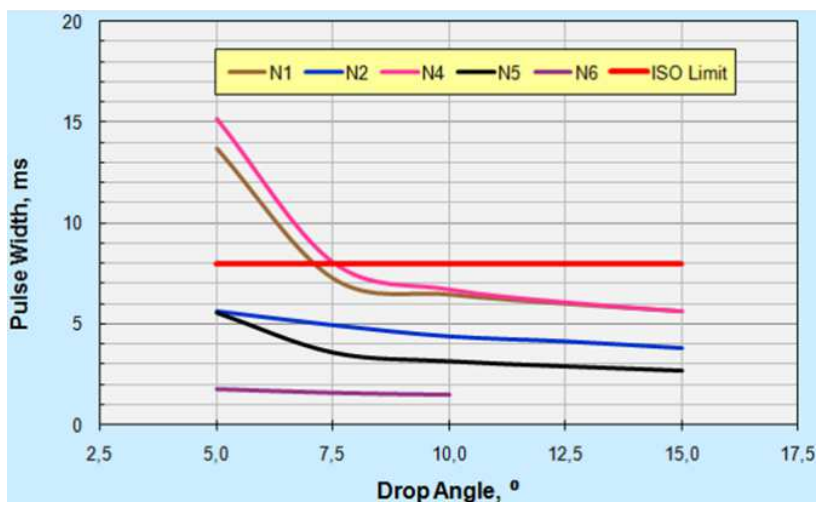

Fig. 6. Pulse width of Ref. transducer and signal conditioner vs. drop angle for samples N1-N6.

- High pulse amplitudes and also very short pulse widths were obtained from the sample N6, teflon (PTFE), which has the highest modulus of elasticity, compared to the other samples used in this investigation.

- Pulse amplitudes show quadratic dependence on the drop angle for all samples (refer to Fig. 5). Pulse limit given in ISO $16063-22$ standard is $8 \mathrm{~ms}$. 


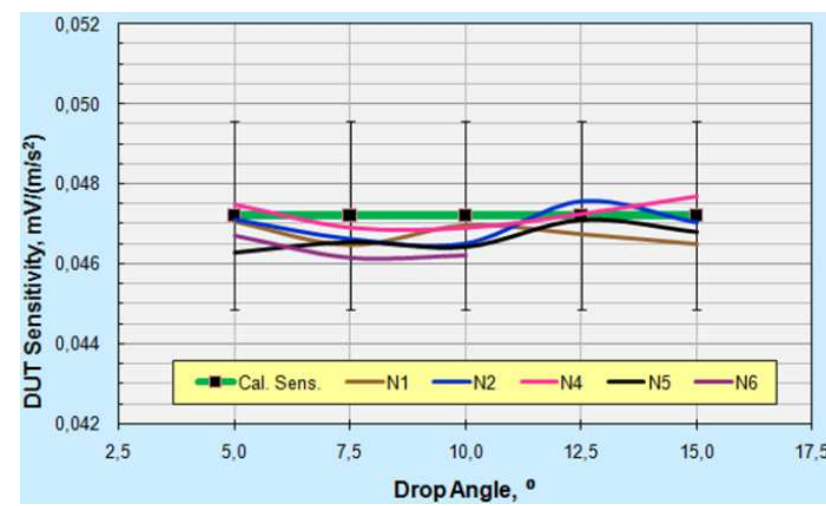

Fig. 7. Calculated shock sensitivity of DUT vs. drop angle, obtained from shock calibrator device, designed in TÜBITAK UME, for samples N1-N6. Green strait line shows the sensitivity values from the sensor manufacturer. The horizontal bars show the uncertainty values in $k=2, \mathrm{k}$ is the coverage factor for the confidence level of $95 \%$.

- All samples fulfill the pulse width requirement for drop angles, starting from $10^{\circ}$ (refer to Fig. 6). Samples N2, N5 and N6 are good choices, due to high pulse amplitudes and also due to small pulse widths.

- All shock sensitivity values of DUT, calculated for drop angles varying from $5^{\circ}$ to $15^{\circ}$ and for the samples N1-N6 show good consistency with the sensitivity value declared in calibration certificate of the reference, and these values are within the uncertainty limits for the calibrations (refer to Fig. 7).

- These results show the equivalence and also the quality of the shock calibrator device designed in TÜBİTAK UME.

\section{Conclusions}

Pendulum shock calibrator, operating in accordance with the pendulum principle was designed, manufactured and installed in TÜBİTAK UME. In order to investigate pulse width and pulse amplitude, generated when hammer pendulum hits the anvil pendulum, different kinds of pad material were used. The modulus of elasticity of the pad materials was obtained, with the regression coefficient larger than 0.995, in Force Laboratory in TUBİTAK UME.

These pads were mounted on the pendulum shock calibrator and then shock pulses were recorded as the output of the reference shock transducer - signal conditioner chain for different drop angles of hammer pendulum. Recorded pulse shapes were analyzed in accordance to the ISO 16063-22 standard, by using a calculation code that was developed in Matlab in TÜBİTAK UME. Dependences of shock amplitude and pulse width on the drop angle and on pad material were investigated. It was found that pulse width decreases with the increase of drop angle, thus resulting in an increase of pulse amplitude.

Secondary level shock calibrations were performed by pendulum shock calibrator. In this calibration two of reference shock transducers are used in back-to-back configuration. One of these transducers was considered as the reference. The calibration uncertainty for this kind of secondary level system mainly depends on the uncertainty of the reference transducer, signal conditioner characteristics, and accuracy of data acquisition system. The obtained shock sensitivity values were compared to the values in the certificate issued by manufacturer of the reference transducer. It was found that these values correspond to each other, within the calibration uncertainty. As a result, the calibrator was verified by using calibrated shock transducers.

\section{References}

[1] ISO 16063-13, Methods for the calibration of vibration and shock transducers - Part 13: Primary shock calibration using laser interferometry, International Organization for Standardization, Geneva 2001.

[2] ISO 16063-22, Methods for the calibration of vibration and shock transducers - Part 22: Shock calibration by comparison to a reference transducer, International Organization for Standardization, Geneva 2005.

[3] A. Link, H.-J. von Martens, W. Wabinski, Proc. SPIE 3411 3411, 224 (1998).

[4] H.-J. von Martens, Metrologia 36, 357 (1999).

[5] M. Peres, R.D. Sill, A New Solution for Shock and Vibration Calibration of Accelerometers in: www.modalshop.com, CAL LAB, 2007.

[6] M. Kobusch, A. Link, A. Buss, T. Bruns, in: IMEKO 20th TC3, 3rd TC16 and 1st TC22 Int. Conf., Merida, Mexico 2007.

[7] E. Bilgiç, Y. Durgut, Acta Phys. Pol. A 128, B-267 (2015).

[8] H. Nozato, A. Oota, T. Ishigami, T. Usuda, Meas. Sci. Technol. 22, 125109 (2011).

[9] Yu-Chung Huang, Jiun-Kai Chen, Hsin-Chia Ho, Chung-Sheng Tu, Chao-Jung Chen, Measurement 45, 2383 (2012).

[10] H. Nozato, T. Bruns, H. Volkers, A. Oota, Meas. Sci. Technol. 25, 035005 (2014).

[11] www.bipm.org, CCAUV.V-K4, Technical Protocol of $C C A U V$ Key Comparison. 